\title{
Synthesis of High Directivity Beams for Conformal Sources
}

\author{
Giovanni Leone, Member, IEEE, Fortuna Munno, and Raffaele Solimene.
}

\begin{abstract}
The role of the source geometry in the the radiation of focusing beams by conformal antennas is examined by the comparison of their directivity functions at different maximum directions. An inverse source problem approach is adopted, where solutions stable with respect to data uncertainties are to be found by relying on the analysis of the pertinent operator by the Singular Values Decomposition. This general framework allows to connect the mean value of the mazimum directivity function to the Number of Degrees of Freedom of the conformal source, which depends only on its electrical length. For each source geometry the focusing far field ensuring the maximum directivity for every pointing direction and the corresponding source current are obtained. The comparison with the ones achieved by the usual phase compensation technique of the source current reveals their optimal behavior. The usefulness of the approach as a tool in antenna synthesis is shown by comparing different geometries for those applications where identical beams are required to be rdiated for the coverage of a larga angular domain.
\end{abstract}

Index Terms - Antenna pattern synthesis, Conformal antennas, Electromagnetic scattering inverse problems, Inverse source problem, Number of Degrees of Freedom, Singular Value Decomposition.

\section{INTRODUCTION}

$\mathrm{C}$ ONFORMAL antennas are attracting large interest for several applications because of their mechanical and electrical advantages. The feature of following the shape of a surface make their aero dynamic behavior suitable for vessels and aircrafts. In addition, the latitude provided by a further geometry dimension makes their radiated field pattern more flexible. For instance, a hemispherical coverage with identical scanning beams cannot be achieved with a planar source due to the inherent pattern degradation far from the broadside direction. On the contrary, a spherical source can guarantee to achieve such kind of requirements.

Unfortunately, a general synthesis method for conformal antennas is lacking, since all approaches start from a prefixed surface definition. Amongst the most common approaches we might mention the ones based on: the alternating reverse projection method [1], the constrained least square approaches [2], [3], the numerical optimization of nonquadratic cost functional by stochastic methods [4]-[7], ad hoc semi-analytical method [8]

However, no general method to compare different source geometries according to their radiation properties seems to have been developed yet. Such a method would be very useful in an early stage of an antenna synthesis problem to steer the design choice in the trade-off between mechanical and electrical performances. In [9], [10] an inverse problem approach is applied to examine the radiation properties of different conformal sources. It is based on the evaluation of the Number of Degrees of Freedom (NDF) and the Point Spread Function (PSF) of the relevant radiation operator. They result from the spectral decomposition of the linear operator connecting the source current to its radiated far field, i.e. the Singular Values Decomposition (SVD). In particular, the NDF defines the dimension of the space of the far field functions that can be radiated by a finite energy source, while the PSF is usually connected to the reconstruction of point-like sources. This approach has revealed itself useful in order to establish a way to compare the general radiation properties of some conformal source [9] before undertaking the synthesis procedure of a particular antenna with defined specifications [11].

However, in some applications, like the above mentioned case of surveillance radar systems with increased angular coverage, the goal of the antenna design is to provide identical beams with the highest directivity. Therefore, it would be fruitful to investigate the possibility of radiating them by different source conformal geometries, by examining not only the corresponding source currents but also the achievable directivity.

The directivity of conformal antennas for beams focusing at different directions has been analyzed starting from the results of the planar case, where a simple geometric relationship holds between the maximum directivity at broadside direction and the one at different directions. This approach is assumed to hold also for other source geometries [12], so that the directivity is related to the size of the 
geometrical projection of the source onto the plane normal to the beam maximum direction [13]. It is apparent that in this way the electrical behavior of the radiating source, which may depend heavily on its shape, is not taken into account,

Therefore, in this paper we mean to discuss and compare the directivity of conformal sources by adopting a different framework, i.e. an inverse problem approach. In particular, in Section II, we outline its mathematical framework, which is based on the SVD of the relevant operators, and recall the features of the solution of the pertinent inverse source problem which are relevant for the analysis to follow concerning highly focusing beams, i.e. the NDF and the PSF. Then, a number of results are derived. In particular, the goals of the paper are: to show that the directivity of the highly focusing beams radiated by a conformal source is connected with its NDF and the PSF of the corresponding radiated field (Section III), and that, in particular, the mean value of the directivity is equal to the NDF; to examine the synthesis problem for maximum directivity beams and examine the corresponding source currents (Section IV), comparing them with the ones commonly adopted on the basis of geometric arguments; to compare the directivity behavior of different source geometries and examine the role of the source element factor (Section V).

\section{ThE MATHEMATICAL BACKGROUND}

In this paper, we discuss the case of a 2D scalar geometry (Fig. 1).

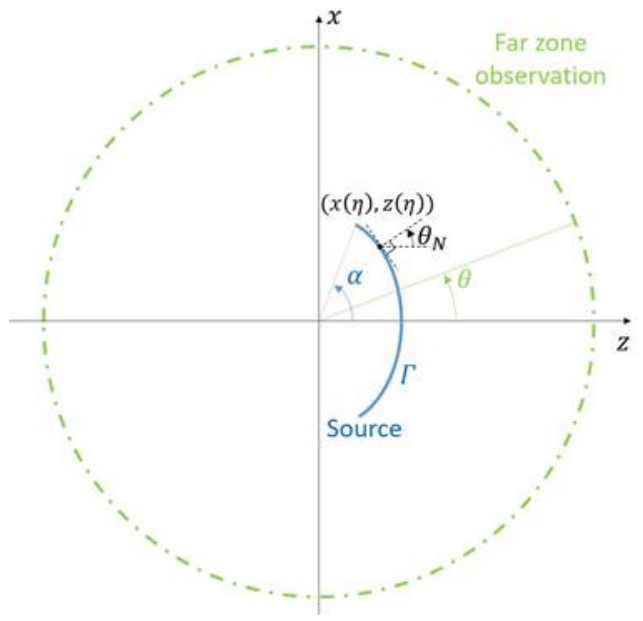

Fig. 1 Relevant to the geometry of theproblem.

An electric scalar surface current $J(\eta)$ is supported over the curve $\Gamma$, where $\eta$ is the variable used to parametrize the curve as $\underline{\gamma}(\eta)=(x(\eta), z(\eta))$. The $J$ source provides a $\mathrm{y}$ oriented electric far field $E(\theta)$ given by the following integral operator

$$
\begin{gathered}
E(\theta)=\int_{\eta_{\min }}^{\eta_{\max }} J(\eta) h(\theta, \eta) e^{j \beta[x(\eta) \sin \theta+z(\eta) \cos \theta]} \\
\left\|\underline{\gamma}^{\prime}(\eta)\right\| d \eta
\end{gathered}
$$

where $\theta \in[-\pi, \pi]$ is the observation variable, $\left\|\underline{\gamma}^{\prime}(\eta)\right\| d \eta$ is the differential element of $\Gamma, \beta=2 \pi / \lambda$ is the free space wavenumber and $\lambda$ is the wavelength. As in [10], [14], the weight function $h(\theta, \eta)$ is meant to add a directive behavior to each source element defined by the $\eta$ parametric variable. In particular, if $\theta_{N}$ is the direction orthogonal to the $\eta$ curve element, the function

$h\left(\theta-\theta_{N}(\eta)\right)=\left(1+\cos \left(\theta-\theta_{N}(\eta)\right)\right)^{m}$,

is chosen so providing a single lobe radiation with the maximum at $\theta_{N}$ while the positive integer $m$ establishes its directive behavior. Therefore, such a function acts as an element factor for the continuous source case.

Because of (1), the vector space of the current functions is mapped to the one of radiated far fields by the operator

$\mathcal{A}: J \in L_{\left[\eta_{\min }, \eta_{\max }\right]}^{2} \rightarrow E \in L_{[-\pi, \pi]}^{2}$,

where, with no prior information beyond the physical feasibility, the vector spaces are assumed as the finite-energy function spaces supported over the range specified in the square brackets and denoted by $L_{[\cdot]}^{2}$.

For each geometry, since $\mathcal{A}$ is compact, it is possible to introduce the SVD of the operator. It consists of the left singular functions $u_{n}(\theta)$ spanning the closure of the operator range, the right singular functions $v_{n}(\eta)$ spanning the operator domain and the singular values $\sigma_{n}$. Since the radiation operator is compact and defined between Hilbert spaces, because of the Riesz-Schauder theorem [15], the singular values belong to a discrete set with no limit points except 0 . In addition, because the kernel of the operator is of exponential type, they exhibit a typical exponential decay after a threshold value. Accordingly, the number of significant singular values provides the NDF of the source. It is usually evaluated as the index in correspondence of the beginning of the exponential decay of the singular values, where the slope of the logarithmic curve changes abruptly. This parameter represents the first important figure of merit to be considered since it provides the dimension of both the space of the current functions that can be stably reconstructed and the one of the corresponding patterns that can be radiated.

The point spread function (PSF) is another parameter useful to the subsequent discussion. When referred to the observation domain, it can be recast as

$\operatorname{PSF}\left(\theta, \theta_{0}\right)=\mathcal{A} \mathcal{A}^{-1} \delta\left(\theta^{\prime}-\theta_{0}\right)$

where $\mathcal{A}^{-1}$ is the regularized inverse of the radiation operator and $\delta\left(\theta^{\prime}-\theta_{0}\right)$ represents the impulsive function centered in $\theta_{0}$. Therefore, it provides the actual field that can be radiated by a source so to approximate an ideal $\delta$-like patter pointing at the $\theta_{0}$ direction.

By resorting to the TSVD algorithm for the inversion, one 
obtains

$\operatorname{PSF}\left(\theta, \theta_{0}\right)=\sum_{n=1}^{N D F} u_{n}(\theta) u_{n}^{*}\left(\theta_{0}\right)$.

The PSF main lobe amplitude gives a quantitative measure of the source capability to radiate a focusing beam at the direction $\theta_{0}$. Generally speaking, it changes for each maximum direction $\theta_{0}$ and depends on the source geometry. However, when its behavior is nearly independent on $\theta_{0}$, the corresponding source can satisfy the requirement of providing uniform coverage within an angular sector by identical beams.

It is apparent that the SVD of (1) is a crucial tool to characterize the properties of the radiation operator and, therefore, the radiation properties of a certain source, but, unfortunately, it is not always known in closed form, except for a circumference source. In all the other cases, in this paper, its numerical computation is performed by densely discretizing source and observation domains and by decomposing the resulting matrix.

\section{NDF AND DIRECTIVITY}

In this section, we examine the connection between the SVD of the radiation operator and the maximum achievable directivity. In particular, the role of the NDF of the source in directivity evaluation is emphasized by employing the left singular functions of the SVD. In [16] a similar, but less general, approach about the directivity analysis is presented but it refers to a set of basis functions obtained by the GramSchmidt procedure

For a $2 \mathrm{D}$ geometry, the directivity at the $\theta_{0}$ direction can be defined as

$D\left(\theta_{0}\right)=\frac{\left|E\left(\theta_{0}\right)\right|^{2}}{\frac{1}{2 \pi} \int_{-\pi}^{\pi}|E(\theta)|^{2} d \theta}$.

If one recasts the radiated field in terms of the left singular functions $u_{n}(\theta)$, that is,

$E(\theta)=\sum_{n=1}^{N D F} c_{n} u_{n}(\theta)$

where $c_{n}=\left\langle E(\theta), u_{n}(\theta)\right\rangle$ and $\langle\cdot, \cdot\rangle$ stands for the scalar product within the appropriate space, eq. (6) becomes

$D\left(\theta_{0}\right)=\frac{\left|\sum_{n=1}^{N D F} c_{n} u_{n}\left(\theta_{0}\right)\right|^{2}}{\frac{1}{2 \pi} \sum_{n=1}^{N D F} c_{n} \sum_{m=1}^{N D F} c_{m}^{*} \int_{-\pi}^{\pi} u_{n}(\theta) u_{m}^{*}(\theta) d \theta}$

The orthonormality property of the singular functions allows writing

$D\left(\theta_{0}\right)=\frac{\left|\sum_{n=1}^{N D F} c_{n} u_{n}\left(\theta_{0}\right)\right|^{2}}{\frac{1}{2 \pi} \sum_{n=1}^{N D F}\left|c_{n}\right|^{2}}$.

The application of the Cauchy-Schwarz inequality to the numerator returns

$$
\begin{aligned}
& D\left(\theta_{0}\right) \leq \frac{\sum_{n=1}^{N D F}\left|c_{n}\right|^{2} \sum_{m=1}^{N D F}\left|u_{m}\left(\theta_{0}\right)\right|^{2}}{\frac{1}{2 \pi} \sum_{n=1}^{N D}\left|c_{n}\right|^{2}}= \\
& =2 \pi \sum_{m=1}^{N D F}\left|u_{m}\left(\theta_{0}\right)\right|^{2}=\mathrm{D}_{\max }\left(\theta_{0}\right)
\end{aligned} .
$$

However, comparison with (5) reveals that this upper bound can be rewritten directly in terms of the PSF as

$D_{\max }\left(\theta_{0}\right)=2 \pi \operatorname{PSF}\left(\theta_{0}, \theta_{0}\right)$.

Let us, now, derive a connection between the maximum directivity $D_{\max }\left(\theta_{0}\right)$ and NDF. We start by considering the mean value of the maximum directivity by varying the focusing angle, that is

$\overline{D_{\max }}=\frac{1}{2 \pi} \int_{-\pi}^{\pi} D_{\max }\left(\theta_{0}\right) d \theta_{0}$.

By (11) it can be cast in terms of the PSF as

$$
\begin{gathered}
\overline{D_{\max }}=\int_{-\pi}^{\pi} \operatorname{PSF}\left(\theta_{0}, \theta_{0}\right) d \theta_{0}= \\
=\int_{-\pi}^{\pi} \sum_{\mathrm{n}=1}^{N D F}\left|u_{\mathrm{n}}\left(\theta_{0}\right)\right|^{2} d \theta_{0}
\end{gathered}
$$

By interchanging integral and sum operators and exploiting the orthonormality of the singular functions, finally, the number of terms of summation (5) is obtained, that is

$\overline{D_{\max }}=N D F$.

Therefore, for all high directivity patterns, the mean directivity value obtained when the focus angle changes is exactly equal to the NDF.

It is important to point out that, as shown in [9], the NDF of a conformal 2D curve source is related only to its electrical length. Therefore, it is apparent that also the directivity is proportional to it, as well known for the linear case. Then, in order to increase the mean directivity value, a longer source is usually required at a fixed frequency.

Alternatively, in order to increase $\overline{D_{\max }}$ without changing the source geometry, some degree of superdirectivity behavior must be accepted. In fact, it is necessary to increase the number of terms of summation (5) beyond NDF, i.e. the dimension of the space of far fields that can be radiated by the source. However, although this leads to more directive patterns, the corresponding source currents suffer from the stability problem, i.e. very different current functions correspond to similar far field patterns.

Finally, from (11) it is clear that, in order to achieve a maximum directivity independent on $\theta_{0}$, the peak of the PSF must be independent on $\theta_{0}$, too. In turn, a sufficient condition to achieve such result is $\left|u_{n}(\theta)\right|$ does not depend on $\theta$. Then

$D_{\max }\left(\theta_{0}\right)=N D F, \forall \theta_{0}$ 
This happens, for example, when the singular functions are complex exponentials, as for the circumference case.

\section{Synthesis of High Directivity Beams}

The patterns and the source currents corresponding to maximum directivity beams pointing at different focusing directions are now highlighted.

In fact, the upper bound in (10) is achieved for each focusing direction $\theta_{0}$ when the coefficients of the radiated field expansion (7) are provided by

$c_{n}=u_{n}^{*}\left(\theta_{0}\right), n=1, \ldots, N D F$,

Accordingly, the field assuring the maximum possible directivity at a fixed focusing angle $\theta_{0}$, for an arbitrary source geometry, is

$\hat{E}(\theta)=\sum_{n=1}^{N D F} u_{n}^{*}\left(\theta_{0}\right) u_{n}(\theta)$,

which is exactly the PSF introduced in (5).

To determine the source corresponding to each radiated field $E(\theta)$, the truncated SVD (TSVD) algorithm is exploited to provide a regularized solution: it consists of retaining only the terms corresponding to the highest singular values in the singular functions expansion of the generalized solution, as

$J(\eta)=\sum_{n=1}^{N D F} \frac{<E(\theta), u_{n}(\theta)>}{\sigma_{n}} v_{n}(\eta)$,

As far as the current corresponding to the field (17) ensuring a focusing beam with the highest possible directivity in the $\theta_{0}$ direction, by substitution of (17) in (18), the following closed form expression for the source current is reached

$\hat{J}(\eta)=\sum_{n=1}^{N D F} \frac{u_{n}^{*}\left(\theta_{0}\right)}{\sigma_{n}} v_{n}(\eta)$

It can be pointed out that a superdirective behavior, as above mentioned, would imply to include more terms in (19), so the corresponding current source becomes more complex to be synthesized. In addition, since the further contributions are related to singular values of smaller level, the unavoidable uncertainties in the practical realization provide unstable current results.

On the other hand, a typical rule-of-thumb to achieve a beam focusing at some direction $\theta_{0}$ in conformal antenna design aims at compensating the different path lengths experienced by the source current elements while propagating to a reference plane orthogonal to maximum direction [17]. In this way the focusing behavior is ensured by the condition that the equivalent sources over this reference plane share approximately the same constant phase. Although improved approaches to define the source phase function exist to synthesize high directivity beams of array antennas [18], in the following we consider the simple phase compensation method for the sake of comparison.

As far as the modulus of this kind of current is concerned, a constant value is commonly adopted so that the current function assumed to provide a focusing beam can be written as

$$
\tilde{J}(\eta)=C\left(\theta_{0}\right) e^{-j \beta\left[x(\eta) \sin \theta_{0}+z(\eta) \cos \theta_{0}\right]},
$$

and the corresponding far field is

$$
\begin{gathered}
\tilde{E}(\theta)=C\left(\theta_{0}\right) \int_{\eta_{\min }}^{\eta_{\max }} h(\theta, \eta) \\
e^{j \beta\left[x(\eta)\left(\sin \theta-\sin \theta_{0}\right)+z(\eta)\left(\cos \theta-\cos \theta_{0}\right)\right]}\left\|\underline{\gamma}^{\prime}(\eta)\right\| d \eta
\end{gathered}
$$

The constant $C\left(\theta_{0}\right)$ is introduced in (20) to guarantee that $\|\hat{E}(\theta)\|=\|\tilde{E}(\theta)\|$ so that the radiated power of both sources is equal.

\section{COMPARISON OF GEOMETRIES}

In the following, we apply the results of the previous Sections and compare numerical and, when available, analytical results of high directivity beams for different source supports. For each geometry the high directivity beam (17), the one obtained by the source current (20), and the approximate directivity prediction $D_{e f f}\left(\theta_{0}\right)$ derived from the rectilinear case [12], [13] by an effective length $l_{\text {eff }}\left(\theta_{0}\right)$ equal to the source projection onto the line orthogonal to the maximum beam direction $\theta_{0}$ (see Appendix) are considered and compared. In addition, the role of the element factor (2) is appreciated.

In order to highlight only the role of the source geometry, we set all sources to the same $20 \lambda$ length, so that a common value of $\mathrm{NDF}=41$ is obtained and $\overline{D_{\max }}=16.13 \mathrm{~dB}$ for all cases.

\section{A. Circumference source}

For the circumference source of radius $R, \eta=\theta, \theta_{N}(\eta)=$ $\theta$ and $\left\|\underline{\gamma}^{\prime}(\eta)\right\| d \eta=R d \phi$ the analytical SVD can be computed in closed form [19]. To be precise, since observation and source spaces coincide (the angular support is the same), in this subsection we refer to eigenvalues $\lambda_{v}$ and eigenfunctions $u_{v}$, the latter spanning both the source and field spaces. It can be found [14] that the eigenvalues have the following expression

$\lambda_{v}=2^{-m+1} \pi R \sum_{k=0}^{2 m}\left(\begin{array}{c}2 m \\ k\end{array}\right) j^{v+k-m} J_{v+k-m}(\beta R)$,

whereas the eigenfunctions are the Fourier harmonics

$u_{v}(\theta)=\frac{e^{j v \theta}}{\sqrt{2 \pi}}$

and $v=-N, \ldots, N, N=\lfloor\beta R\rfloor$, where $\lfloor\cdot\rfloor$ returns the 
nearest integer less than or equal to its argument. Furthermore, independently on $m$, the NDF is equal to

$N D F \approx 2\lfloor\beta R\rfloor+1$.

In addition, (15) holds as the eigenfunctions (23) satisfy the pertinent condition, i.e. their modulus is constant with respect to $\theta$.

Hence, the field to be radiated assuring high directivity is

$\hat{E}(\theta)=\sum_{n=-N}^{N} \frac{e^{j n\left(\theta-\theta_{0}\right)}}{2 \pi}=\frac{2 N+1}{2 \pi} \frac{\sin \left[(2 N+1)\left(\theta-\theta_{0}\right) / 2\right]}{\sin \left[\left(\theta-\theta_{0}\right) / 2\right]}$,

whereas the corresponding current function can be found by (19) as

$\hat{J}(\phi)=\sum_{n=-N}^{N} \frac{e^{j n\left(\phi-\theta_{0}\right)}}{2 \pi \lambda_{n}}$.

On the other side, the current function (20) becomes

$\tilde{J}(\phi)=C e^{-j \beta R \cos \left(\theta_{0}-\phi\right)}$,

which provides the radiated fields

$$
\begin{gathered}
\tilde{E}(\theta)=\pi C R 2^{-2 m+1} \sum_{k=0}^{2 m}\left(\begin{array}{c}
2 m \\
k
\end{array}\right) e^{j(m-k) \theta} \\
J_{m-k}\left(2 \beta R \sin \left(\frac{\theta-\theta_{0}}{2}\right)\right) e^{-j(m-k)\left(\frac{\theta+\theta_{0}}{2}\right)}
\end{gathered}
$$

A numerical example confirms that the directivity of the field (25) is higher than the one of the field (28). Fig. 2 shows both the fields radiated by a circumference source with $R=$ $3.18 \lambda$ when $m=0$ for $\theta_{0}=0$ : the directivity of (28) is $14.53 \mathrm{~dB}$, lower than the one of (25), as expected; in addition, the $\hat{E}$ field guarantees side lobes lower than those of $\tilde{E}$ by 6 $\mathrm{dB}$.
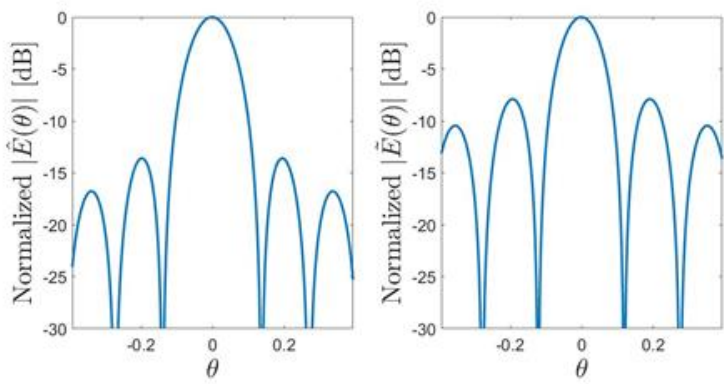

Fig. 2 Comparison of the normalized amplitude of the field $\hat{E}$ (left panel) and $\tilde{E}$ (right panel) when $m=0$.

In Fig. 3, the corresponding source currents are compared. It can be appreciated that the current function (27) is very different from the optimal one.

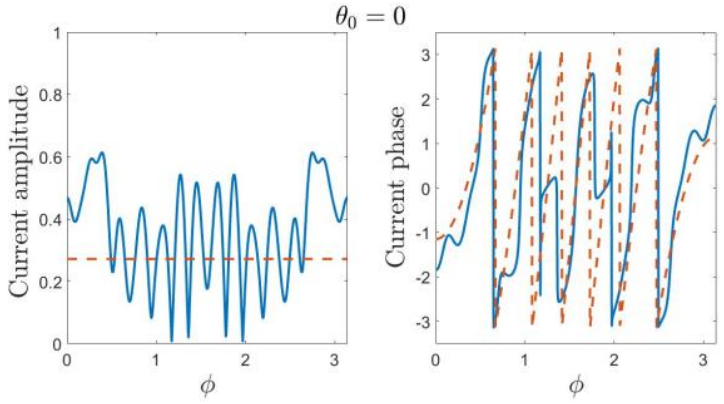

Fig. 3 Comparison of the amplitude and phase of the currents $\hat{J}(x)$ (solid blue line) and $\tilde{J}(x)$ (dashed red line) corresponding to far field $\hat{E}$ and $\tilde{E}$ of Fig. $2(C=0.27)$. For symmetry reasons, only the interval $[0, \pi]$ is reported.

Similar results still hold for a different choice of $m$ in the weight function (2), accounting for a more directive behavior of the current elements. For $m=2$, the fields $\hat{E}$ and $\tilde{E}$ are shown in Fig. 4 while the corresponding sources are shown in Fig. 5. Again, the directivity of the field $\widetilde{E}$, i.e. $13.68 \mathrm{~dB}$, is lower than the one of $\widehat{E}$ while the side lobes are higher by $2 \mathrm{~dB}$.
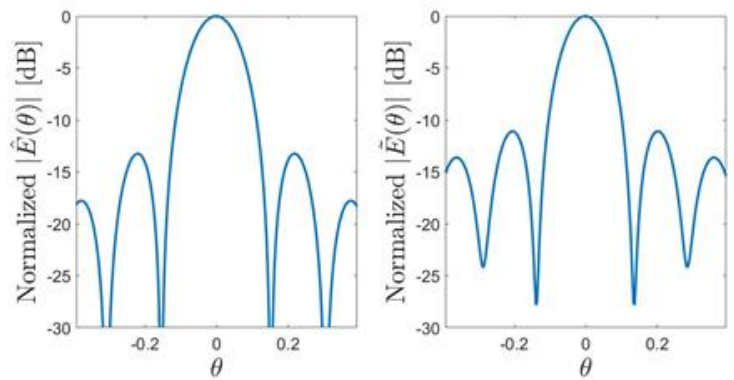

Fig. 4 Comparison of the normalized amplitude of the field $\hat{E}$ (left panel) and $\tilde{E}$ (right panel) when $m=2$.

Fig. 5 shows that, in order to radiate the high directivity field, the amplitude current profile exhibits a selective behavior for those source elements closer to the main beam direction $\theta_{0}=0$ due to the assumed directive behavior of the element factor, while, now, its phase function can be well approximated by the path length compensation behavior in (20). On the whole, the current function (27) is still different from the optimal one only for the amplitude.

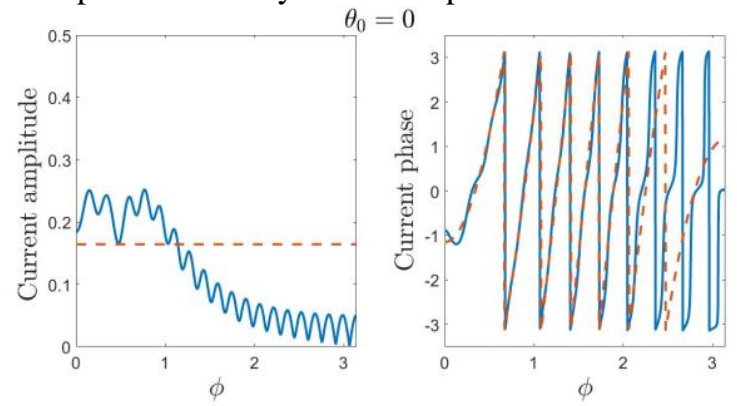

Fig. 5 Comparison of the amplitude and phase of the currents $\hat{J}(x)$ (solid blue line) and $\tilde{J}(x)$ (dashed red line) corresponding to far field $\hat{E}$ and $\tilde{E}$ of Fig. $4(C=0.16)$. For symmetry reasons, only the interval $[0, \pi]$ is reported.

\section{B. Rectilinear source}

For an $x$-aligned rectilinear source $(z(\eta)=0)$ the current is recast as a function of the $x$ coordinate and 
$\left\|\underline{\gamma}^{\prime}(\eta)\right\| d \eta=d x$. Accordingly, (1) becomes

$E(\theta)=(1+\cos (\theta))^{m} \int_{-a}^{a} J(x) e^{j \beta x \sin \theta} d x$,

where $2 a$ is the length of the source. The weight function introduces a directive behavior towards the broadside direction when $m>0$ but, as can be seen from Fig. 6, does not affect the number of significant singular values (the NDF), which are those preceding the steep decay.

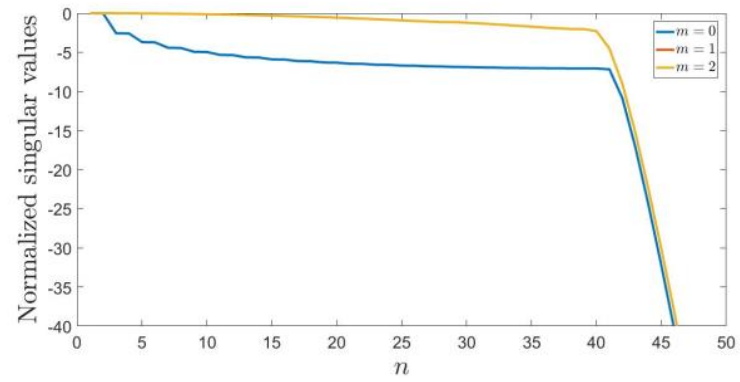

Fig. 6 Normalized singular values of the operator (1) for $m=0,2$ when the source is a rectilinear source with $a=10 \lambda$.

For this operator, an analytical SVD is not available and the numerically computed one is employed.

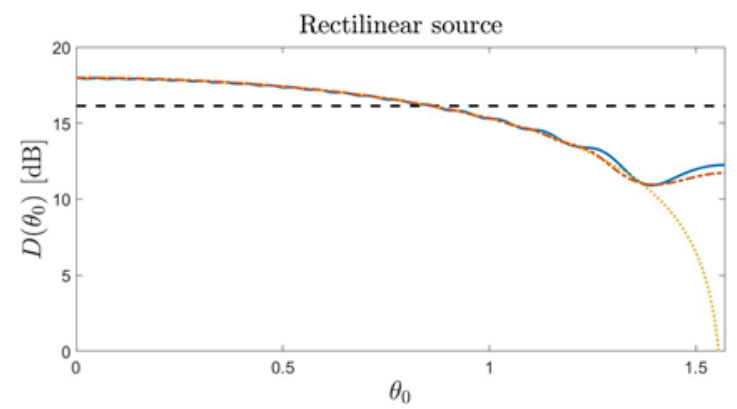

Fig. 7 Behavior of the maximum directivity $D_{\max }\left(\theta_{0}\right)$ (solid blue line), the directivity of $\tilde{E}$ (dash-dotted red line) and $D_{\text {eff }}\left(\theta_{0}\right)$ (dotted yellow line) for a rectilinear source with $a=10 \lambda$ when $m=0$. For symmetry reasons, only the interval $[0, \pi / 2]$ is reported. The dashed black line represents the value of $\overline{D_{\max }}$

For the case $m=0$, the maximum directivity (11) is computed for all focusing angle (Fig. 7) when the high directivity fields are provided by (17). As expected by a rectilinear source, better focusing performances are reached near the broadside direction than any other direction, as confirmed by Fig. 7 where the $D_{\max }\left(\theta_{0}\right)$ is higher than (14) around $\theta_{0}=0$.
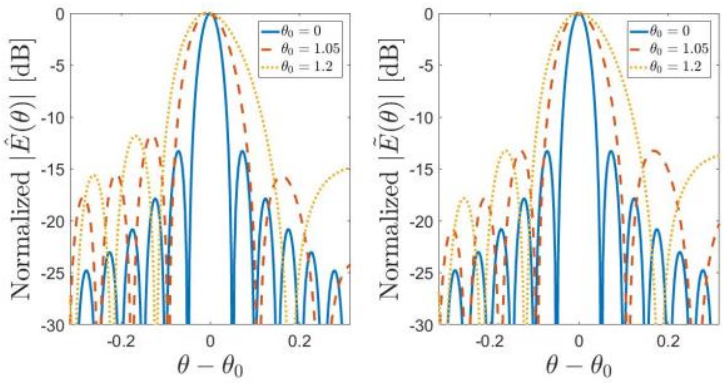

Fig. 8 Comparison of the normalized amplitude of the field $\hat{E}$ (left panel) and $\tilde{E}$ (right panel) for focusing angles $\theta_{0}=0,1.05,1.57 \mathrm{rad}$ and when $m=$

The source current corresponding to (20) for this geometry reads as

$\tilde{J}(x)=C\left(\theta_{0}\right) e^{-j \beta x \sin \theta_{0}}$,

and the field radiated by this source can be easily computed by (21) as

$\tilde{E}(\theta)=C\left(\theta_{0}\right) 2 a \operatorname{sinc}\left[\beta a\left(\sin \theta-\sin \theta_{0}\right)\right]$,

The comparison of the directivities of the $\tilde{E}(\theta)$ and $\hat{E}(\theta)$ fields of Fig. 7 reveals that, in most circumstances the differences between them are very small. This means that, with a reasonable small error, the field (31) belongs to the space spanned by the pertinent singular functions and can be expanded as in (17). Thus, it can be concluded that the patterns returned by (31) are very close to the ones with the highest directivity and, accordingly, it can be expected that the same occurs also for the corresponding sources. This is confirmed by Figs. 8 and 9 .

For the same reason and because of its very definition which is referred to a linear source, it can be expected that $D_{e f f}\left(\theta_{0}\right)$, as defined in Appendix, provides the maximum directivity, too, at least for $\theta_{0}$ not too close to $\pi / 2$.

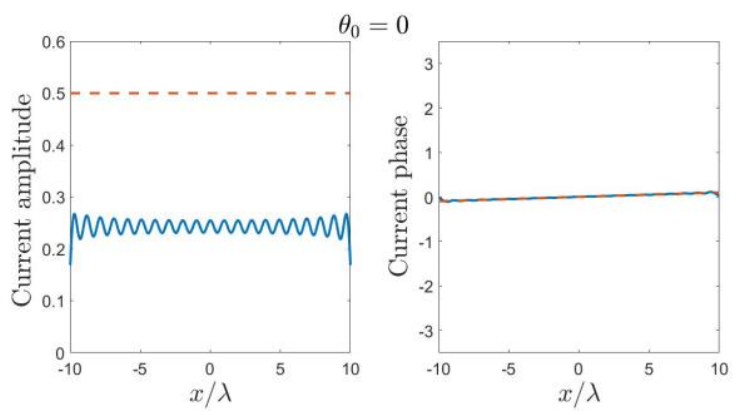



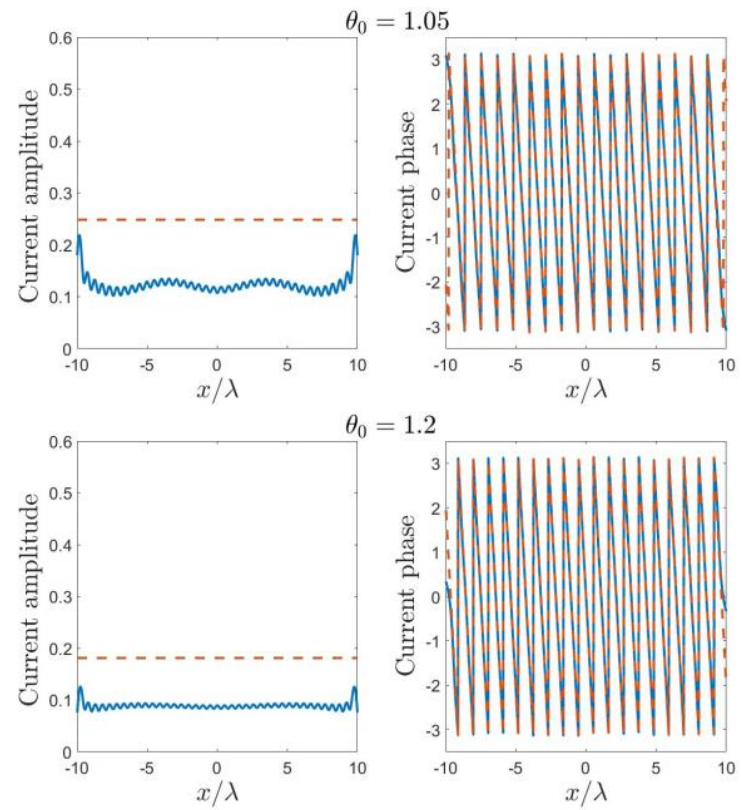

Fig. 9 Comparison of the amplitude and phase of the currents $\hat{J}(x)$ (solid blue curve) and $\tilde{J}(x)$ (dashed red curve) corresponding to far field $\hat{E}$ and $\tilde{E}$ of Fig. $8(C(0)=0.5, C(1.05)=0.25, C(1.2)=0.18)$.

\section{Semi-circumference}

For an arc of circumference of radius $R, \eta=\phi, \theta_{N}(\eta)=$ $\theta, \quad\left\|\underline{\gamma}^{\prime}(\eta)\right\| d \eta=R d \phi, \quad \eta_{\min }=-\alpha, \quad \eta_{\max }=\alpha$ in the integral operator (1), but, differently from the circumference case, no analytical results for the pertinent SVD are available in this case. Some asymptotic reasonings [9], [20] have led to an estimation of the NDF as

$N D F \approx 2\left\lfloor\frac{\beta R \alpha}{\pi}\right\rfloor+1$,

but this is the only analytical result that can be exploited in this analysis. So, the SVD of (1) is numerically computed, the field and the sources assuring the maximum directivity are built by (17) and (19), and the comparison source is provided by (20) leading to the field (21)

$$
\begin{gathered}
\tilde{E}(\theta)=\alpha C\left(\theta_{0}\right) R 2^{-2 m+1} \sum_{k=0}^{2 m}\left(\begin{array}{c}
2 m \\
k
\end{array}\right) e^{j(m-k) \theta} \\
\sum_{p=-\infty}^{+\infty} J_{p}\left(2 \beta R \sin \left(\frac{\theta-\theta_{0}}{2}\right)\right) e^{-j p \frac{\theta+\theta_{0}}{2}} \\
\operatorname{sinc}[(p-m+k) \alpha]
\end{gathered}
$$

which can be derived as in [14].

Fig. 10 presents the maximum directivity, the directivity of $\tilde{E}$ and $D_{e f f}\left(\theta_{0}\right)$, when $l_{e f f}\left(\theta_{0}\right)=R\left(1+\left|\cos \theta_{0}\right|\right)$ (see Appendix), with respect to the focusing angle for a semicircumference source $(\alpha=\pi)$ with $\mathrm{R}=6.37 \lambda$ (corresponding to $\mathrm{NDF}=41$ ) and $m=0$. Looking at the maximum directivity curve, the highest values are reached around 0 and $\pi$ focusing directions, while the lowest ones are around $\theta_{0}= \pm \pi / 2$, but its deviation from the mean value is around $\pm 1 \mathrm{~dB}$. The directivities of the $\tilde{E}$ fields are always lower than those of $\hat{E}$ and also below $\overline{D_{\max }}$; the geometrical approach, instead, underestimates the actual capability of the source to radiate similar beams at different directions and predicts dirictivity values $1 \mathrm{~dB}$ lower than the optimal ones.

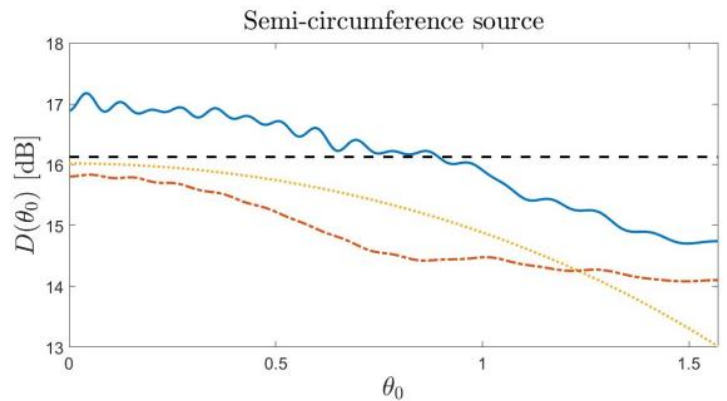

Fig. 10 Behavior of the maximum directivity $D_{\max }\left(\theta_{0}\right)$ (solid blue line), the directivity of $\tilde{E}$ (dash-dotted red line) and the one geometrically predicted (dotted yellow line) for a semi-circumference source with $R=6.37 \lambda$ when $m=0$. For symmetry reasons, only the interval $[0, \pi / 2]$ is shown. The dashed black line represents the corresponding value of $\overline{D_{\max }}$.

The field $\hat{E}(\theta)$ is compared with $\tilde{E}(\theta)$ in Fig. 11, and the corresponding directivities are reported in Table I. It can be appreciated that the optimal sources guarantee higher directivities for all maximum angles.
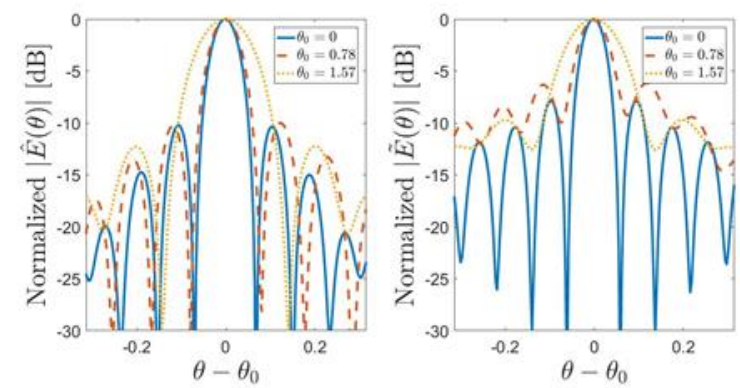

Fig. 11 Comparison of the normalized amplitude of the field $\hat{E}$ (left panel) and $\tilde{E}$ (right panel), for a semi-circumference source with $R=6.37 \lambda$ when $m=0$ and $\theta_{0}=0, \pi / 4, \pi / 2$.

In Fig. 12 the corresponding source currents are compared and the $|\hat{J}(\phi)|$ functions are far from being uniform, while the phase functions are approximated by the path compensating ones of (20).

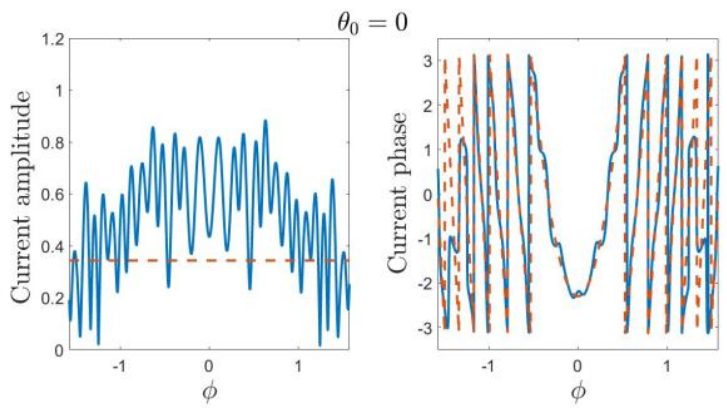




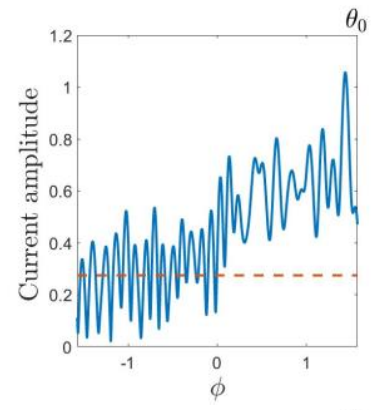

$=0.78$
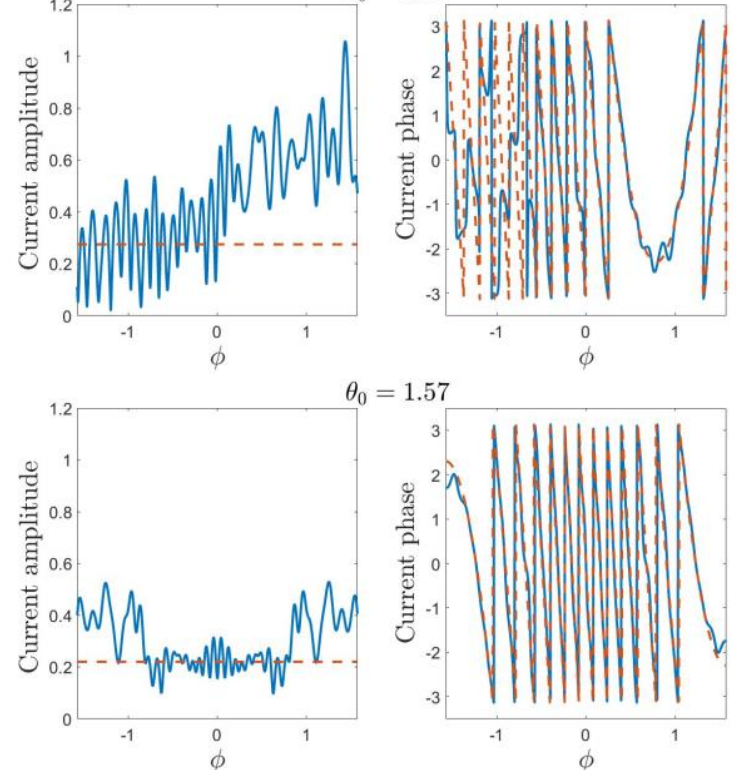

$\theta_{0}=1.57$

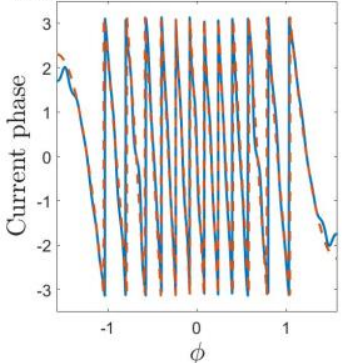

Fig. 12 Comparison of the amplitude and phase of the currents $\hat{J}(x)$ (solid blue line) and $\tilde{J}(x)$ (dashed red line) corresponding to far field $\hat{E}$ and $\tilde{E}$ of Fig. 11, respectively $(C(0)=0.34, C(0.78)=0.27, C(1.57)=0.22)$.

TABLE I

DIRECTIVITIES OF THE $\hat{E}$ AND $\tilde{E}$ FIELDS FOR A SEMI-CIRCUMFERENCE SOURCE WITH $R=6.37 \Lambda$ WHEN $m=0$.

\begin{tabular}{lccc}
\hline \hline \multicolumn{1}{c}{$\theta_{0}$} & $\begin{array}{c}D\left(\theta_{0}\right) \\
{[\mathrm{dB}]}\end{array}$ & of $\hat{E}$ & $\begin{array}{c}D\left(\theta_{0}\right) \\
{[\mathrm{dB}]}\end{array}$ \\
\hline $0 \mathrm{rad}$ & 16.89 & 15.81 & \\
$0.78 \mathrm{rad}$ & 16.22 & 14.53 \\
$1.57 \mathrm{rad}$ & 14.74 & 14.10 \\
\hline \hline
\end{tabular}

The same results shown in Figs. 10-12 and in Table I are presented in Figs. 13-15 and in Table II for the case $m=2$. One can notice that the presence of the weight function increases the dynamics of the maximum directivity: the peak value of the curve is emphasized ( $19.09 \mathrm{~dB}$ instead of 16.89 $\mathrm{dB})$, but now the focusing capabilities of the source are significant only in a single half-space, as the maximum directivities reduce for $\theta_{0}>\pi / 2$.

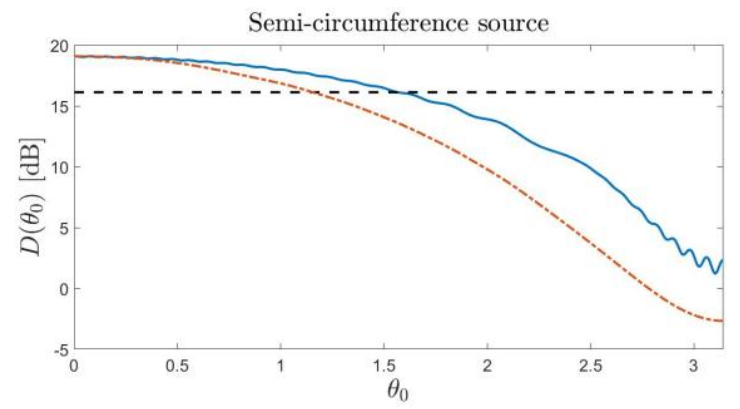

Fig. 13 Behavior of the maximum directivity $\mathrm{D}_{\max }\left(\theta_{0}\right)$ (solid blue line) and the directivity of $\widetilde{E}$ (dash-dotted red line) for a semi-circumference source with $R=6.37 \lambda$ and $m=2$. For symmetry reasons, only the interval $[0, \pi]$ is reported. The dashed black line represents the value of $\overline{\mathrm{D}_{\max }}$.

Fig. 14 shows the role of the source element pattern in achieving focusing patterns with side lobes level lower than the case $m=0$. Finally, Fig. 18 reveals that, again, the amplitude of the $\hat{J}(\phi)$ functions are far from uniformity, while the phase functions are approximated by the path compensating ones of (20). In addition, as the main beam scans away from the broadside direction, only a part of the source current provides the main contribution to the radiated field.
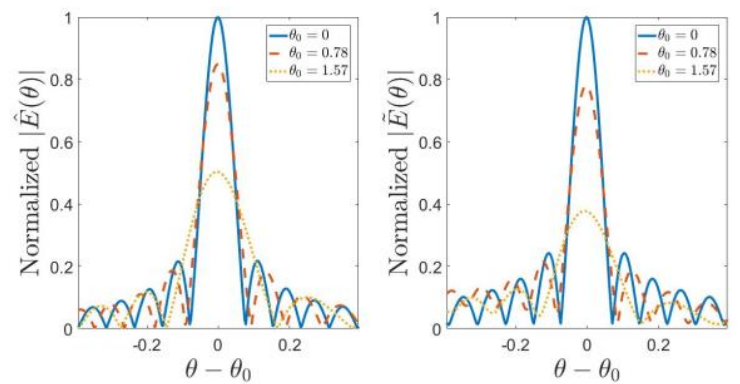

Fig. 14 Comparison of the normalized amplitude of the field $\hat{E}$ (left panel) and $\tilde{E}$ (right panel) for a semi-circumference source with $R=6.37 \lambda$ when $m=2$ and $\theta_{0}=0, \pi / 4, \pi / 2$.
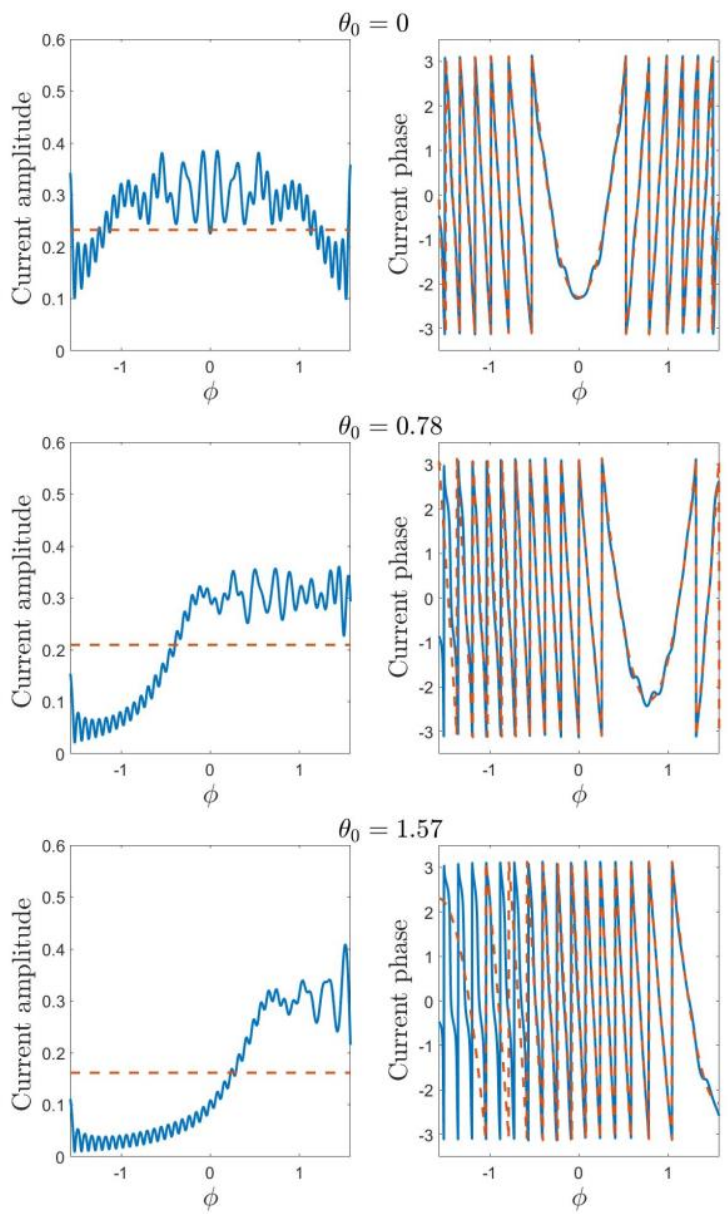

Fig. 15 Comparison of the amplitude and phase of the currents $\hat{J}(x)$ (solid blue line) and $\tilde{J}(x)$ (dashed red line) corresponding to far field $\hat{E}$ and $\tilde{E}$ of Fig. 17, respectively $(C(0)=0.23, C(0.78)=0.21, C(1.57)=0.16)$. 
TABLE II

DIRECTIVITIES OF THE $\hat{E}$ AND $\tilde{E}$ FIELDS FOR A SEMI-CIRCUMFERENCE SOURCE WITH $R=6.37 \Lambda$ WHEN $m=2$.

\begin{tabular}{lll}
\hline \hline \multicolumn{1}{c}{$\theta_{0}$} & $\begin{array}{c}D\left(\theta_{0}\right) \text { of } \hat{E} \\
{[\mathrm{~dB}]}\end{array}$ & $\begin{array}{c}D\left(\theta_{0}\right) \text { of } \tilde{E} \\
{[\mathrm{~dB}]}\end{array}$ \\
\hline $0 \mathrm{rad}$ & 19.09 & 19.08 \\
$0.78 \mathrm{rad}$ & 18.38 & 17.64 \\
$1.57 \mathrm{rad}$ & 16.11 & 13.58 \\
\hline \hline
\end{tabular}

\section{Parabolic arc}

We consider now a parabolic arc with semi-latus rectum $p$ so that the center of the reference system coincides with its focus. The curve can be parameterized with respect to the $\eta=\phi$ angle as

$\underline{\gamma}(\phi):\left\{\begin{array}{l}x(\phi)=r(\phi) \sin \phi \\ z(\phi)=r(\phi) \cos \phi\end{array}\right.$,

for $\phi \in[-\alpha, \alpha]$ and $\alpha<\pi / 2$, where

$r(\phi)=\frac{p}{1+\cos (\phi)}$

Then, the radiation operator (1) writes

$$
\begin{gathered}
E(\theta)=\int_{-\alpha}^{\alpha} J(\phi) h\left(\theta-\theta_{N}(\phi)\right) \\
e^{j \beta r(\phi) \cos (\theta-\phi)}\left\|\underline{\gamma}^{\prime}(\phi)\right\| d \phi
\end{gathered}
$$

where the differential element can be computed as

$\left\|\underline{\gamma}^{\prime}(\phi)\right\|=r(\phi) \sqrt{1+\left(\frac{\sin \phi}{1+\cos \phi}\right)^{2}}$,

and

$\theta_{N}(\phi)=\operatorname{atan}\left(\frac{\sin \phi}{1+\cos (\phi)}\right)$

The focusing source $\tilde{J}$, instead, particularizes as

$$
\tilde{J}(\phi)=C\left(\theta_{0}\right) e^{-j \beta r(\phi) \cos \left(\theta_{0}-\phi\right)} .
$$

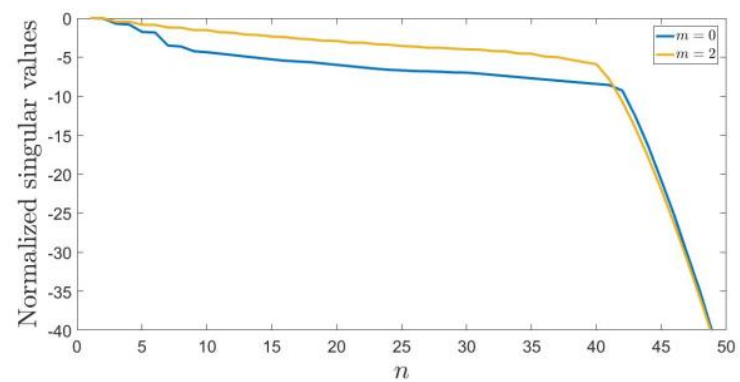

Fig. 16 Normalized singular values of the operator (1) with $m=0,2$ for a parabolic arc with $p=8.58 \lambda$ and $\alpha=\pi / 2$.

For any choice of $m$ it can be found that the NDF is given approximately by [9]
$N D F \approx\left\lfloor\frac{2 L}{\lambda}\right\rfloor$,

where $L$ is the length of the arc. This is confirmed by the results of the numerical SVD of (1) shown in Fig. 16, referring to a parabolic arc with $\alpha=\pi / 2, p=8.58 \lambda$ and, accordingly, $L=19.7 \lambda$ so that $N D F \simeq 41$.

A comparison between Figs. 17-18 and Figs. 19-20 and between Tables III and IV reveals not only that $\widehat{E}$ can assure directivities higher than $\tilde{E}$, as expected, but also that the choice $m=2$ allows reaching higher directivities than $m=$ 0 , both in the broadside direction and in a wider angular range around it. In particular, $D\left(\theta_{0}\right)>\overline{D_{\max }}$ nearly for $\theta_{0}>\pi / 2$ when $m=2$, due to the directive effect of the element factor, so allowing to achieve a quite full hemispherical coverage. In addition, for $m=0 D_{e f f}\left(\theta_{0}\right)$ (see Appendix) returns an estimation lower than the maximum value achievable (Fig. 17).

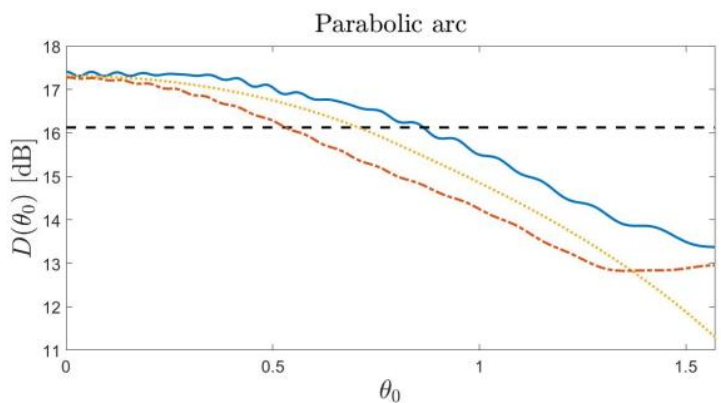

Fig. 17 Behavior of the maximum directivity $D_{\max }\left(\theta_{0}\right)$ (solid blue line), the directivity of $\tilde{E}$ (dash-dotted red line) and $D_{\text {eff }}\left(\theta_{0}\right)$ (dotted yellow line) of a parabolic source with $\alpha=\pi / 2$ and $p=8.58 \lambda$ when $m=0$. For symmetry reasons, only the interval $[0, \pi / 2]$ is shown. The dashed black line represents the corresponding value of $\overline{D_{\max }}$.
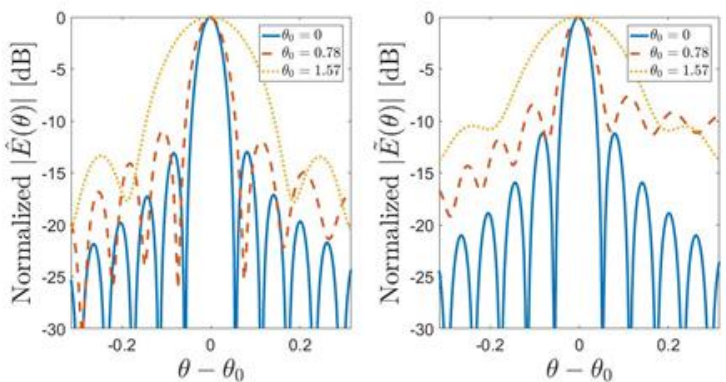

Fig. 18 Comparison of the normalized amplitude of the field $\hat{E}$ (left panel) and $\tilde{E}$ (right panel) for a parabolic source with $\alpha=\pi / 2$ and $p=8.58 \lambda$ when $m=0$ and $\theta_{0}=0, \pi / 4, \pi / 2$. 


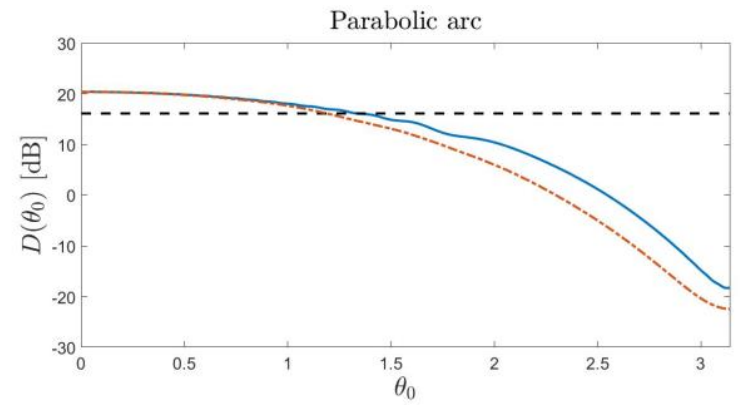

Fig. 19 Behavior of the maximum directivity $D_{\max }\left(\theta_{0}\right)$ (solid blue line) and the directivity of $\tilde{E}$ (dash-dotted red line) of a parabolic source with $\alpha=$ $\pi / 2$ and $p=8.58 \lambda$ when $m=2$. For symmetry reasons, only the interval $[0, \pi]$ is shown. The dashed black line represents the corresponding value of $\overline{D_{\max }}$.
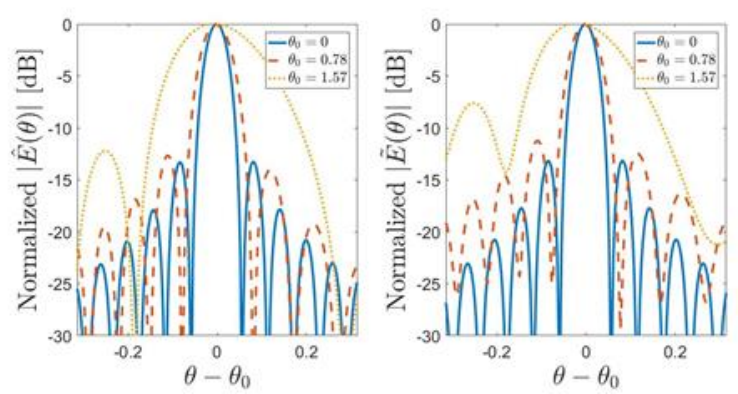

Fig. 20 Comparison of the normalized amplitude of the field $\hat{E}$ (left panel) and $\tilde{E}$ (right panel) for a parabolic source with $\alpha=\pi / 2$ and $p=8.58 \lambda$ when $m=2$ and $\theta_{0}=0, \pi / 4, \pi / 2$.

TABLE III

DIRECTIVITIES OF THE $\hat{E}$ AND $\tilde{E}$ FIELDS FOR A PARABOLIC ARC WITH $\alpha=$

\begin{tabular}{lcc}
\multicolumn{3}{c}{$\pi / 2$ AND $p=8.58 \lambda$ wHEN $m=0}$. \\
\hline \hline$\theta_{0}$ & $D\left(\theta_{0}\right)$ of $\hat{E}$ & $D\left(\theta_{0}\right)$ of $\tilde{E}$ \\
& {$[\mathrm{~dB}]$} & {$[\mathrm{dB}]$} \\
\hline $0 \mathrm{rad}$ & 17.41 & 17.28 \\
$0.78 \mathrm{rad}$ & 16.37 & 15.08 \\
$1.3 \mathrm{rad}$ & 13.38 & 12.95 \\
\hline \hline
\end{tabular}

TABLE IV

DiRECTIVITIES OF THE $\hat{E}$ AND $\tilde{E}$ FIELDS FOR A PARABOLIC ARC WITH $\alpha=$

\begin{tabular}{lcc}
\multicolumn{3}{c}{$\pi / 2$ AND $p=8.58 \lambda$ wHEN $m=2}$. \\
\hline \hline$\theta_{0}$ & $\begin{array}{c}D\left(\theta_{0}\right) \text { of } \hat{E} \\
{[\mathrm{~dB}]}\end{array}$ & $\begin{array}{c}D\left(\theta_{0}\right) \text { of } \tilde{E} \\
{[\mathrm{~dB}]}\end{array}$ \\
\hline $0 \mathrm{rad}$ & 20.40 & 20.36 \\
$0.78 \mathrm{rad}$ & 18.95 & 18.79 \\
$1.3 \mathrm{rad}$ & 14.60 & 12.35 \\
\hline \hline
\end{tabular}

Finally, Figs. 21 and 22 show once again that the modulus of the current function of optimal high directivity beams is always different from the constant one, whereas its phase function can be approximated under the path compensation approach and that $|\hat{J}(\phi)|$ may be relevant on limited angular regions of the source according to the chosen focusing angle.
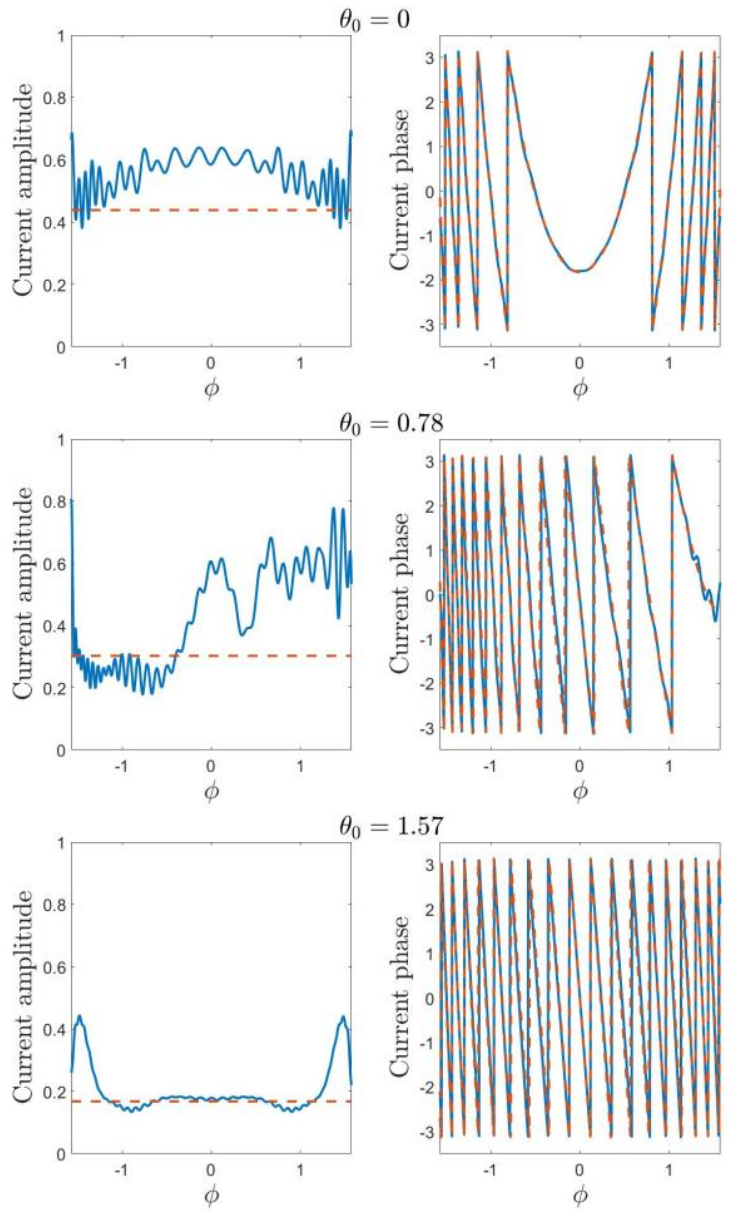

Fig. 21 Comparison of the amplitude and phase of the currents $\hat{J}(x)$ (solid blue line) and $\tilde{J}(x)$ (dashed red line) corresponding to far field $\hat{E}$ and $\tilde{E}$ of Fig. 18, respectively $(\mathrm{C}(0)=0.44, \mathrm{C}(0.78)=0.3, \mathrm{C}(1.57)=0.17)$.
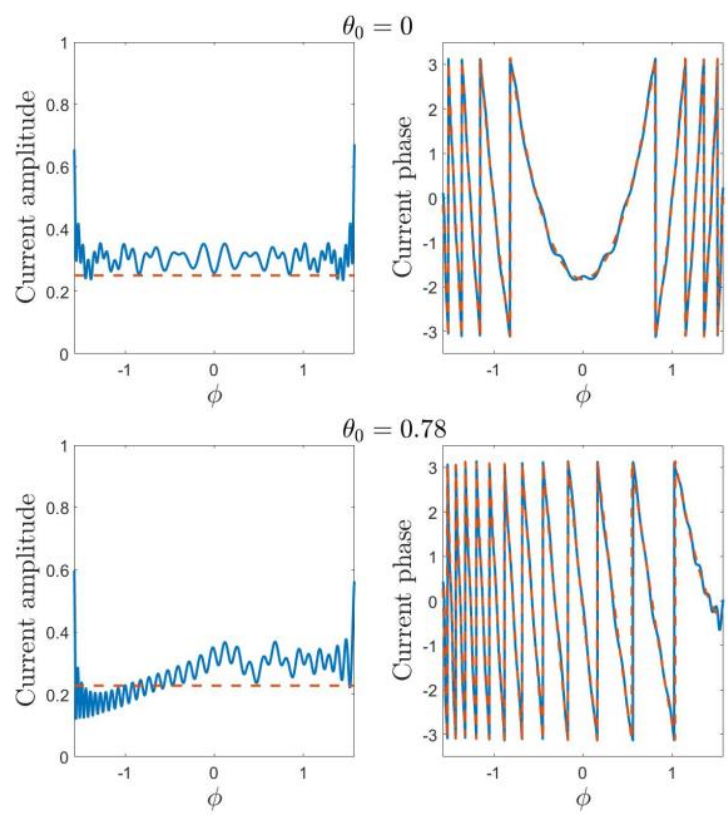

$\theta_{0}=0.78$

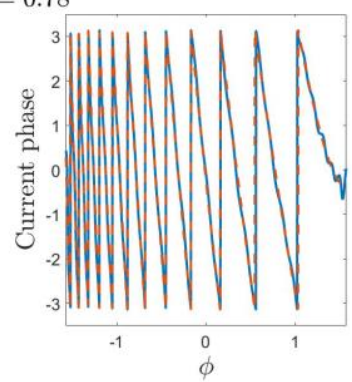




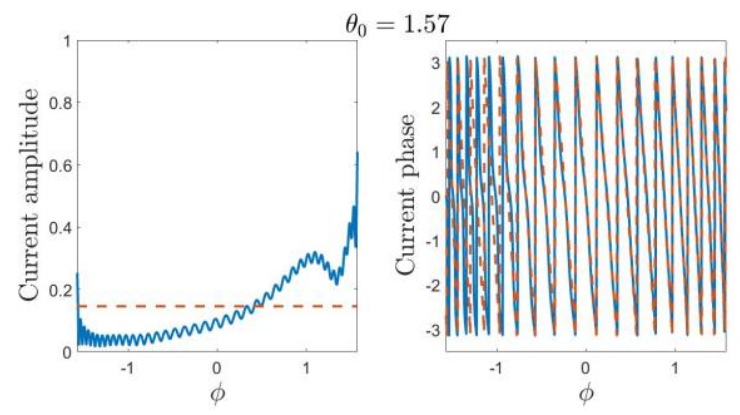

Fig. 112 Comparison of the amplitude and phase of the currents $\hat{J}(x)$ (solid blue line) and $\tilde{J}(x)$ (dashed red line) corresponding to far field $\hat{E}$ and $\tilde{E}$ of Fig. 20, respectively $(\mathrm{C}(0)=0.25, \mathrm{C}(0.78)=0.23, \mathrm{C}(1.57)=0.14)$.

\section{E. Angle source}

We refer to the angle source consisting of two linear sources arranged under an angle shape as in Fig. 26, with $\phi_{02}=360^{\circ}-\phi_{01}$, the middle point of each side satisfying $x_{02}=-x_{01}$ and $z_{01}=z_{02}$ and the same length $a_{2}=a_{1}=$ a.

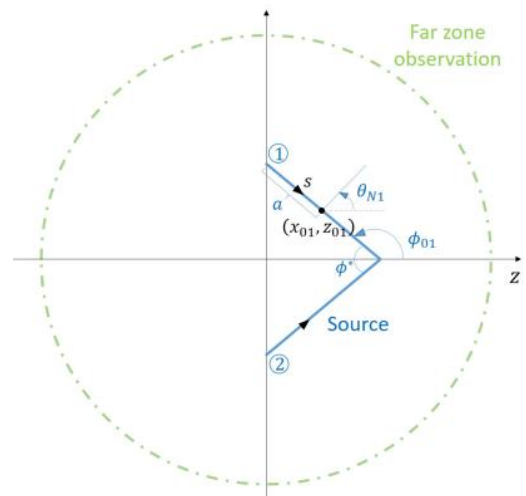

Fig. 123 Angle source geometry

The two rectilinear sources support the electric currents $J_{1}$ and $J_{2}$ which give rise to the following field

$E(\theta)=\mathcal{A}_{1} J_{1}+\mathcal{A}_{2} J_{2}$,

With

$\mathcal{A}_{i} J_{i}=\int_{-\mathrm{a}}^{\mathrm{a}} J_{i}(s) h\left(\theta-\theta_{N i}\right)$
$e^{j \beta\left[x_{i}(s) \sin \theta+z_{i}(s) \cos \theta\right]} d s$

for $i=1,2$, where $s$ represents the arc length variable, $x_{i}(s)=x_{0 i}-s \sin \phi_{0 i}$ and $z_{i}(s)=z_{0 i}-s \cos \phi_{0 i}$ are the rectilinear coordinates of the source points expressed as a function of the arc length $s \in[-a, a]$, and $\theta_{N i}=\phi_{0 i}+$ $(-1)^{i} \frac{\pi}{2}$ is the angle providing the direction orthogonal to each segment. In [10], it has been found that, when dealing with sets of non-overlapping rectilinear sources, an approximation of the NDF is given by the sum of the NDF due to a single rectilinear source. Hence, in this case,

$N D F \approx 2\left\lfloor\frac{4 a}{\lambda}\right\rfloor$, while the angle assuring an omnidirectional coverage is $\phi_{01}=2.68 \mathrm{rad}$, which is the one used in the following.

For the examples below, the value of $a$ is chosen equal to $5 \lambda$ so that $N D F \simeq 41$. When $m=0$ (Fig. 24 ), the maximum achievable directivity assumes almost the same value over the all angular interval, as expected, slightly fluctuating around the main value of $16.13 \mathrm{~dB}$. As far as $D_{e f f}\left(\theta_{0}\right)$ is concerned (see Appendix), also in this case it underestimates the highest directivity attainable by the source (at least $2 \mathrm{~dB}$ ). When $m=2$ instead (Fig. 25) higher values of the maximum directivity can be reached, but they remain high only on part of the domain: the maximum directivity falls below the mean value for $\left|\theta_{0}\right|>1.98 \mathrm{rad}$, implying that a high directivity coverage is possible only on a part of the observation domain. However, a hemispherical coverage is guaranteed, which can be useful in some radar applications.

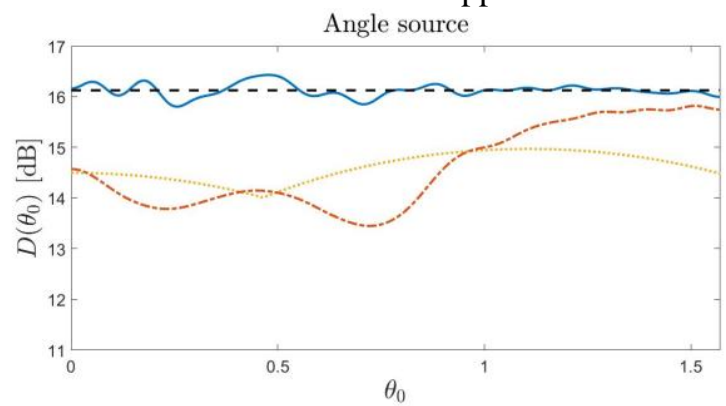

Fig. 24 Behavior of the maximum directivity $D_{\max }\left(\theta_{0}\right)$ (solid blue line), the directivity of $\tilde{E}$ (dash-dotted red line) and $D_{\text {eff }}\left(\theta_{0}\right)$ (dotted yellow line) of an angle source with $a=5 \lambda$ and $\phi_{01}=2.68 \mathrm{rad}$ when $m=0$. For symmetry reasons, only the interval $[0, \pi / 2]$ is shown. The dashed black line represents the corresponding value of $\overline{D_{\max }}$.

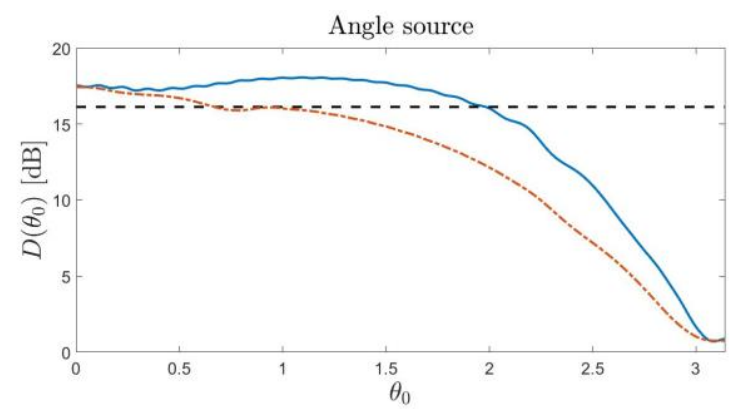

Fig. 25 Behavior of the maximum directivity $D_{\max }\left(\theta_{0}\right)$ (solid blue line) and the directivity of $\tilde{E}$ (dash-dotted red line) of an angle source with $a=5 \lambda$ and $\phi_{01}=2.68 \mathrm{rad}$ when $m=2$. For symmetry reasons, only the interval $[0, \pi]$ is shown. The dashed black line represents the corresponding value of $\overline{D_{\max }}$.

As expected, $\hat{E}$ assures directivities higher than $\tilde{E}$, regardless of $m$, as it can be observed from Tables V and VI and from Figs. 24-27. 

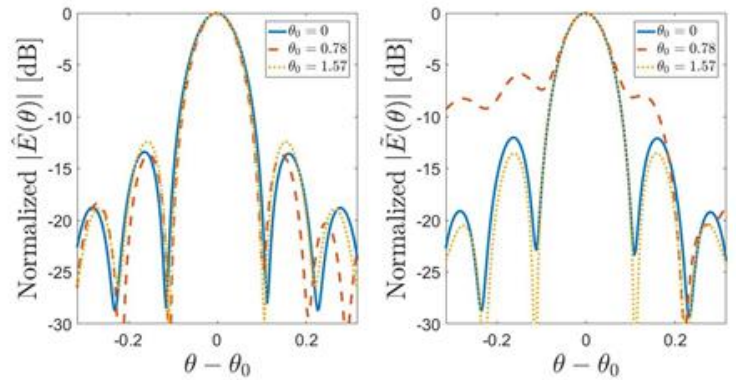

Fig. 136 Comparison of the normalized amplitude of the field $\hat{E}$ (left panel) and $\tilde{E}$ (right panel) for an angle source with $a=5 \lambda$ and $\phi_{01}=2.68$, when $m=0$ and $\theta_{0}=0, \pi / 4, \pi / 2$.
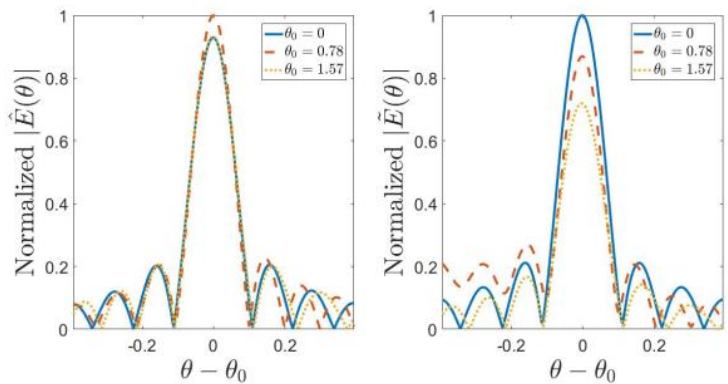

Fig. 27 Comparison of the normalized amplitude of the field $\hat{E}$ (left panel) and $\tilde{E}$ (right panel) for an angle source with $a=5 \lambda$ and $\phi_{01}=2.68$, when $m=2$ and $\theta_{0}=0, \pi / 4, \pi / 2$.

TABLE V

DiRECTIVITIES OF THE FoCUSING FIELDS $\hat{E}$ AND $\tilde{E}$ FOR AN ANGLE SOURCE

\begin{tabular}{lcc}
\multicolumn{3}{c}{ WITH $a=5 \lambda, \phi_{01}=2.68$ RAD WHEN $m=0}$. \\
\hline \hline \multicolumn{1}{c}{$\theta_{0}$} & $\begin{array}{c}D\left(\theta_{0}\right) \text { of } \hat{E} \\
{[\mathrm{~dB}]}\end{array}$ & $\begin{array}{c}D\left(\theta_{0}\right) \text { of } \tilde{E} \\
{[\mathrm{~dB}]}\end{array}$ \\
\hline $0 \mathrm{rad}$ & 16.16 & 14.57 \\
$0.78 \mathrm{rad}$ & 16.13 & 13.60 \\
$1.57 \mathrm{rad}$ & 16.00 & 15.75 \\
\hline \hline
\end{tabular}

ABLE VI

DiReCTIVITIES OF THE Focusing FIELDS $\hat{E}$ AND $\tilde{E}$ FOR AN ANGLE SOURCE

\begin{tabular}{lrr}
\multicolumn{3}{c}{ WITH $a=5 \lambda, \phi_{01}=2.68$ RAD WHEN $m=2}$. \\
\hline \hline$\theta_{0}$ & $\begin{array}{r}D\left(\theta_{0}\right) \text { of } \hat{E} \\
{[\mathrm{~dB}]}\end{array}$ & $\begin{array}{r}D\left(\theta_{0}\right) \text { of } \tilde{E} \\
{[\mathrm{~dB}]}\end{array}$ \\
\hline $0 \mathrm{rad}$ & 17.53 & 17.44 \\
$0.78 \mathrm{rad}$ & 17.85 & 15.90 \\
$1.57 \mathrm{rad}$ & 17.55 & 14.55 \\
\hline \hline
\end{tabular}

Finally, the source currents are compared in Figs. 28 and 29 . Both the linear sources contribute in the same way to the radiation of $\hat{E}$ when $\theta_{0}=0$, while for the other cases the line facing the $\theta_{0}$ direction mainly contributes to the radiation. When a weight function is present, the sectorial contribution of each segment is further emphasized.
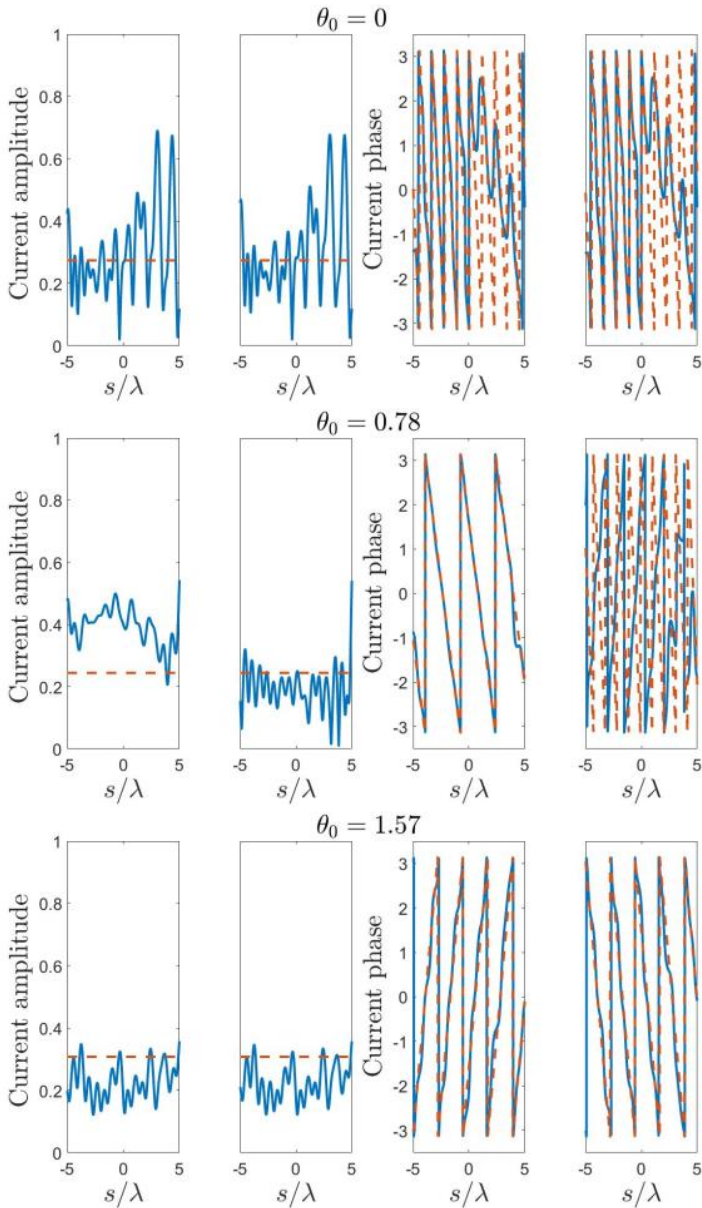

Fig. 28 Comparison of the amplitude and phase of the currents $\hat{j}(x)$ (solid blue curve) and $\tilde{J}(x)$ (dashed red curve) corresponding to far field $\hat{E}$ and $\tilde{E}$ of Fig. 26, respectively $(C(0)=0.27, C(0.78)=0.24, C(1.57)=0.31)$.
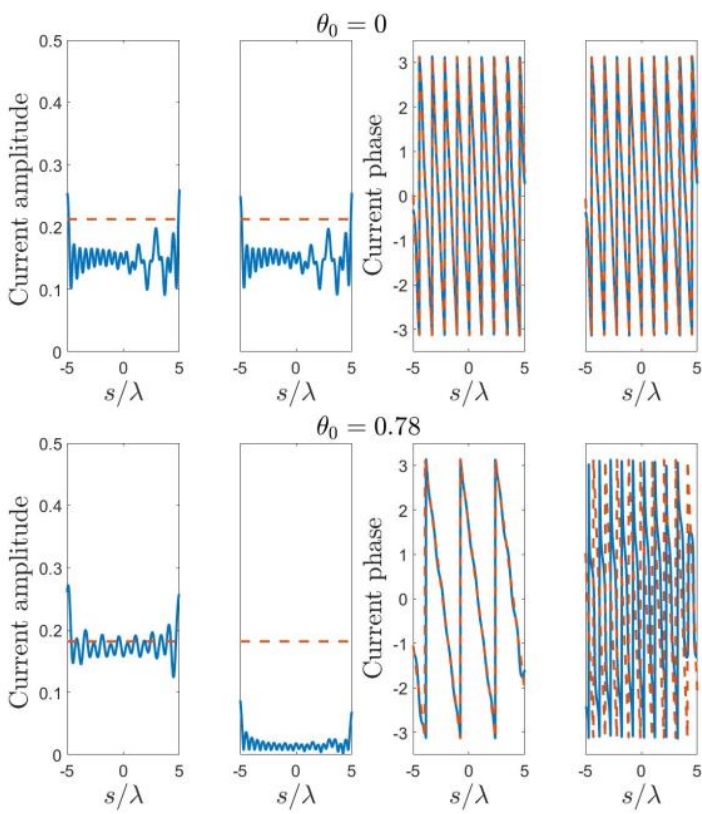


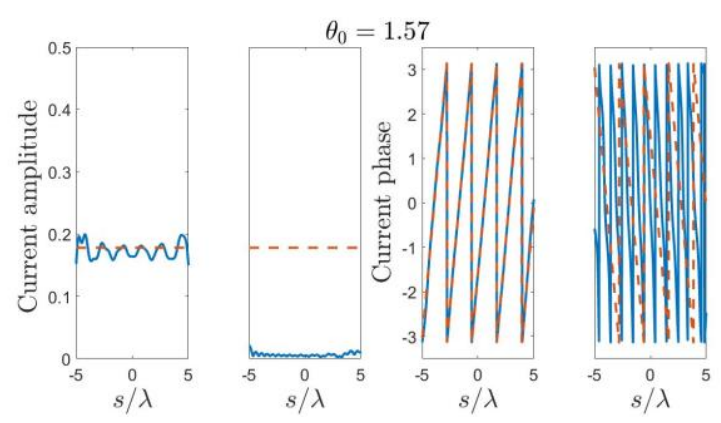

Fig. 29 Comparison of the amplitude and phase of the currents $\hat{J}(x)$ (solid blue curve) and $\tilde{J}(x)$ (dashed red curve) corresponding to far field $\hat{E}$ and $\tilde{E}$ of Fig. 27, respectively $(C(0)=0.21, C(0.78)=0.18, C(1.57)=0.18)$.

\section{Discussion OF the Results AND CONCLUSIONS}

Conformal antennas synthesis lacks for general approaches to compare their radiation behavior, as, for instance, the ability to radiate identical high directivity beams in a wide angular range. By exploiting an inverse problem approach and the concept of the NDF of a source and of the PSF, an upper bound of the directivity has been established. In addition, it is found that the mean value of the directivity is equal to the NDF of the source, independently on its geometry. Since it has been shown that, in turn, the NDF is proportional to the electrical length of the source, it arises that increasing the mean directivity requires longer conformal sources. Thus, sources of different geometry, but with the same length, can radiate fields that have the same mean directivity, although the directivity profiles, for each maximum direction within the observation space, are in general different.

The adopted approach allows to find both the source and the corresponding radiated field for high directivity beams. On the other hand, an usual approximation to achieve a high directivity beam with a conformal source current aims only at compensating the phase delay of the path between each source element and a reference plane normal to the maximum direction, while the current amplitude function is considered constant. Thus, a comparison between these approximate solutions and the optimal high directivity beams reveals that, usually, the phase function of the optimal source current approximates the optimal one quite well. However, the amplitude of the optimal source current is always markedly different from a constant function, so to explain suboptimal directivities achieved by the approximate approach.

In addition the directivity predictions based only on geometrical arguments underestimate always the maximum directivities the source can actually achieve.

Therefore, caution must be exercised in the synthesis of the source current of a conformal antenna for high directivity applications unless resorting to more accurate mathematical modelling of the radiation.

A comparison between different source geometries in achieving uniform beams and directivities within a hemispherical angular sector reveals that the angular and the semi-circumference sources can ensure a uniform directivity behavior over the full hemi-spherical angular range. The parabolic and the rectilinear source, instead, do not allow to radiate focusing high directivity beams in the endfire direction.

The role of a directive element factor can be also appreciated by this approach, since it affects the directivity angular profile, without changing the mean directivity value. In particular, it allows to enhance radiation in the angular domain of interest for a parabolic source, so allowing to achieve both a more uniform behavior and directivity values higher than the mean one.

The whole approach can be extended readily to conformal array antennas as it provides the way to compare different geometrical configurations before undertaking the synthesis procedure.

\section{APPENDIX}

In [12], [13] the peak directive gain of a conformal source whose maximum points at an angle $\theta_{0}$ is assumed proportional to the projection of the source area onto the plane normal to $\theta_{0}$. Following an analogous geometrical approach, one can think to link the directivity of a 2D source to the projection of its length onto the straight line normal to the pointing angle $\theta_{0}$. In this appendix we derive this link for a rectilinear source and then extend the result to a generic $2 \mathrm{D}$ curved source.

Let us consider the case $m=0$ and suppose that the current density that feeds the $2 a$ long linear source is (30) with $C\left(\theta_{0}\right)=1 \forall \theta_{0}$ for sake of simplicity. Then, the directivity is

$D\left(\theta_{0}\right)=\frac{2 \pi}{\int_{-\pi}^{\pi} \sin c^{2}\left[\beta a\left(\sin \theta-\sin \theta_{0}\right)\right] d \theta}$.

Let us focus on the denominator. Taking advantage of the eveness of $\operatorname{sinc}^{2}$, it can be written as

$\int_{-\pi}^{\pi} \operatorname{sinc}^{2}\left[\beta a\left(\sin \theta-\sin \theta_{0}\right)\right] d \theta=$
$2 \int_{-\frac{\pi}{2}}^{\frac{\pi}{2}} \operatorname{sinc}^{2}\left[\beta a\left(\sin \theta-\sin \theta_{0}\right)\right] d \theta$

which, moving to the variable $u=\beta a\left(\sin \theta-\sin \theta_{0}\right)$, becomes

$2 \int_{-\beta a\left(1+\sin \theta_{0}\right)}^{\beta a\left(1-\sin \theta_{0}\right)} \frac{\operatorname{sinc}^{2} u}{\beta a|\cos \theta|} d u$

Since the function to be integrated is nothing else than the square module of a field focusing in the $\theta_{0}$ direction, it will contribute mainly to the integral for the angles close $\theta_{0}$ (i.e., $u \simeq 0$ ). This allows a first approximation to be introduced into the denominator

$2 \int_{-\beta a\left(1+\sin \theta_{0}\right)}^{\beta a\left(1-\sin \theta_{0}\right)} \frac{\operatorname{sinc}^{2} u}{\beta a\left|\cos \theta_{0}\right|} d u$. 
Extending, then, the integration interval between $-\infty$ and $+\infty$ (which holds for $\beta a>>1$ ) and remembering that the integral of $\operatorname{sinc}^{2}$ is known and equal to $\pi$, it can be obtained that

$\int_{-\pi}^{\pi} \operatorname{sinc}^{2}\left[\beta a\left(\sin \theta-\sin \theta_{0}\right)\right] d \theta \approx \frac{2 \pi}{\beta a\left|\cos \theta_{0}\right|}$.

The directivity of the focusing field (31) can, thus, be approximated as

$D\left(\theta_{0}\right) \approx \beta a\left|\cos \theta_{0}\right|=\frac{\pi}{\lambda} 2 a\left|\cos \theta_{0}\right|$.

The directivity is maximum in $\theta_{0}=0$ and equal to $D_{\text {max }}=\beta a$. The factor $2 a\left|\cos \theta_{0}\right|$ geometrically corresponds to the projection of the source onto the line orthogonal to the focusing direction. Hence, an effective length such that

$D_{e f f}\left(\theta_{0}\right) \approx \frac{\pi}{\lambda} l_{e f f}\left(\theta_{0}\right)$

can be introduced. By extending (A.7) to a generic curved source, an approximate criterium to predict the directivity behaviour of the source is provided.

In Table VII the value of $l_{e f f}\left(\theta_{0}\right)$ for the analyzed source shapes is reported.

TABLE VII

EFFECTIVE LENGTH $l_{\text {eff }}\left(\theta_{0}\right)$ FOR SOME SOURCE SHAPES.

\begin{tabular}{cc}
\hline \hline $\begin{array}{c}\text { Source shape } \\
\text { Rect. } \\
\text { Semi-circ. }\end{array}$ & $\begin{array}{c}l_{\text {eff }}\left(\theta_{0}\right) \\
2 a\left|\cos \theta_{0}\right| \\
R\left(1+\left|\cos \theta_{0}\right|\right)\end{array}$ \\
Parab. & $\left\{\begin{array}{c}p\left|\cos \theta_{0}\right|+\sqrt{1+\frac{1}{\tan ^{2} \theta_{0}}}, \quad\left|\theta_{0}\right| \in\left[\frac{\pi}{4}, \frac{3 \pi}{4}\right] \\
2 p\left|\cos \theta_{0}\right|\end{array}\right.$ \\
Angle & $\left\{\begin{array}{c}2 a\left|\sin \left(\phi_{01}-\theta_{0}\right)\right|, \quad \theta_{0} \in\left[\pi-\phi_{01}, \frac{\pi}{2}\right] \cup\left[-\phi_{01},-\frac{\pi}{2}\right] \\
2 a\left|\sin \left(\theta_{0}+\phi_{01}\right)\right|, \quad \theta_{0} \in\left[\frac{\pi}{2}, \phi_{01},\right] \cup\left[-\frac{\pi}{2},-\pi+\phi_{01}\right] \\
4 a\left|\sin \phi_{01} \cos \theta_{0}\right|,\end{array}\right.$ \\
\hline \hline
\end{tabular}

\section{REFERENCES}

[1] R. Karimzadeh, M. Hakkak, A. Haddadi and K. Forooraghi, "Conformal array pattern synthesis using the weighted alternating reverse projection method considering mutual coupling and embedded-element pattern effects", IET Microw. Antennas Propag., vol. 6, no. 6, pp. 621-626, Apr. 2012.

[2] L. I. Vaskelainen, "Constrained least-squares optimization in conformal array antenna synthesis," IEEE Trans. Antennas Propag., vol. 55 , no. 3 , pp. 859-867, 2007.

[3] H. Oraizi, and H. Soleimani, "Optimum pattern synthesis of nonuniform spherical arrays using the Euler rotation," IET Microw. Antennas Propag., vol. 9, no. 9, pp. 898-904, 2015,

[4] J. Ou Yang, Q. R. Yuan, F. Yang, H. J. Zhou, Z. P. Nie, and Z. Q. Zhao, "Synthesis of Conformal Phased Array With Improved NSGAII Algorithm”, IEEE Trans. Antennas Propag, vol. 57, no. 12, pp. 4006-4009, Dec. 2009.

[5] W. T. Li, Y. Q. Hei, and X. W. Shi, "Pattern synthesis of conformal arrays by a modified particle swarm optimization", Progr. Electromagn. Res., vol. 117, pp. 237-252, 2011.

[6] Y. Y. Bai, S. Xiao, C. Liu and B. Z. Wang, "A hybrid IWO/PSO algorithm for pattern synthesis of conformal phased arrays", IEEE Trans. Antennas Propag., vol. 61, no. 4, pp. 2328-2332, Apr. 2013.
[7] R. J. Allard and D. H. Werner, "Radiation pattern synthesis for arrays of conformal antennas mounted on arbitrarily-shaped threedimensional platforms using genetic algorithms," IEEE Trans. Antennas Propag., vol. 51, no. 5, pp. 1054-1062, May 2003.

[8] W. T. Li, X. W. Shi, Y. Q. Hei, S. F. Liu, and J. Zhu, "A Hybrid Optimization Algorithm and Its Application for Conformal Array Pattern Synthesis", IEEE Trans. Antennas Propag., vol. 58, no. 10, pp. 3401-3406, Oct. 2010.

[9] G. Leone, F. Munno and R. Pierri, "Inverse source on conformal conic geometries", IEEE Trans. Antennas Propag., early access, 2020, doi: 10.1109/TAP.2020.3016375.

[10] G. Leone, F. Munno and R. Pierri, "Synthesis of Angle Arrays by the NDF of the Radiation Integral", IEEE Trans. Antennas Propag, early access, 2020, doi: 10.1109/TAP.2020.3026910.

[11] G. Leone, "Source Geometry Optimization for Hemispherical Radiation Pattern Coverage" IEEE Trans. Antennas Propag, vol. 64, no. 5, pp 2033 - 2038 , May 2016.

[12] L. Josefsson, and P. Persson, Conformal array antenna theory and design. vol. 29, John wiley \& sons, 2006.

[13] T. Pelham, G. Hilton, E. Mellios, and R. Lewis, "Predicting Conformal Aperture Gain From 3-D Aperture and Platform Models", IEEE Antennas and Wireless Propagat. Letters, vol 16, pp. 700-703, 2017.

[14] G. Leone, F. Munno and R. Pierri, "Radiation of a Circular Arc Source in a Limited Angle for Non-uniform Conformal Arrays", accepted for publication on IEEE Trans. Antennas Propag., 2020

[15] A. E. Taylor, D. Lay, Introduction to Functional Analysis 2nd Edition, Wiley., 1980.

[16] J. N. Sahalos, Othogonal Methods for Array Synthesis: Theory \& the ORAMA Computer Tool. West Sussex, , U.K. : Wiley, 2006 .

[17] I. Ullah, S. Khattak and B. D. Braaten, "Broadside Pattern Correction Techniques for Conformal Antenna Arrays" in Advances in Array Optimization, ed. E. Aksoy, InTech, doi: 10.5772/intechopen.83276.

[18] H. M. Bernety, S. Venkatesh, and D. Schurig, "Analytical phasing of arbitrarily oriented arrays using a fast, analytical far-field calculation method," IEEE Trans. Antennas Propag., vol. 66, no. 6, pp. 2911-2922, June 2018.

[19] G. Leone, M.A. Maisto, R. Pierri, "Application of Inverse Source Reconstruction to Conformal Antennas Synthesis", IEEE Trans. Antennas Propag, vol. 66, no. 3 pp. 1436 - 1445, Mar. 2018.

[20] G. Leone, M.A. Maisto, R. Pierri, "Inverse Source of Circumference Geometries: SVD Investigation based on Fourier Analysis", Progr. Electromagn. Res. M, vol. 76, pp. 217-230, 2018. 